\title{
The Fekete-Szegö inequality for close-to-convex functions with respect to a certain starlike function dependent on a real parameter
}

Bogumiła Kowalczyk and Adam Lecko²*

*Correspondence:

alecko@matman.uwm.edu.pl ${ }^{2}$ Department of Analysis and

Differential Equations, University of Warmia and Mazury, Słoneczna 54,

Olsztyn, 10-710, Poland

Full list of author information is

available at the end of the article

$$
\begin{aligned}
& \text { Abstract } \\
& \text { Given } \alpha \in[0,1] \text {, let } g_{\alpha}(z):=z /(1-\alpha z)^{2}, z \in \mathbb{D}:=\{z \in \mathbb{C}:|z|<1\} \text {. An analytic standardly } \\
& \text { normalized function } f \text { in } \mathbb{D} \text { is called close-to-convex with respect to } g_{\alpha} \text { if there exists } \\
& \delta \in(-\pi / 2, \pi / 2) \text { such that } \\
& \qquad \operatorname{Re}\left\{\mathrm{e}^{\mathrm{i} \delta} \frac{z f^{\prime}(z)}{g_{\alpha}(z)}\right\}>0, \quad z \in \mathbb{D} .
\end{aligned}
$$

For the class $\mathcal{C}\left(g_{\alpha}\right)$ of all close-to-convex functions with respect to $g_{\alpha}$, the Fekete-Szegö problem is studied.

MSC: Primary 30c45

Keywords: Fekete-Szegö problem; close-to-convex functions; close-to-convex functions with respect to the Koebe function; close-to-convex functions with argument $\delta$; functions convex in the positive direction of the imaginary axis

\section{Introduction}

The classical problem settled by Fekete and Szegö [1] is to find for each $\lambda \in[0,1]$ the maximum value of the coefficient functional

$$
\Phi_{\lambda}(f):=\left|a_{3}-\lambda a_{2}^{2}\right|
$$

over the class $\mathcal{S}$ of univalent functions $f$ in the unit disk $\mathbb{D}:=\{z \in \mathbb{C}:|z|<1\}$ of the form

$$
f(z)=z+\sum_{n=2}^{\infty} a_{n} z^{n}, \quad z \in \mathbb{D}
$$

By applying the Loewner method they proved that

$$
\max _{f \in \mathcal{S}} \Phi_{\lambda}(f)= \begin{cases}1+2 \exp (-2 \lambda /(1-\lambda)), & \lambda \in[0,1) \\ 1, & \lambda=1\end{cases}
$$

O2014 Kowalczyk and Lecko; licensee Springer. This is an Open Access article distributed under the terms of the Creative Commons Attribution License (http://creativecommons.org/licenses/by/2.0), which permits unrestricted use, distribution, and reproduction in any medium, provided the original work is properly cited. 
The problem of calculating $\max _{f \in \mathcal{F}} \Phi_{\lambda}(f)$ for various compact subclasses $\mathcal{F}$ of the class $\mathcal{A}$ of all analytic functions $f$ in $\mathbb{D}$ of the form (1.1), as well as for $\lambda$ being an arbitrary real or complex number, was considered by many authors (see, e.g., [2-10]).

Let $\mathcal{S}^{*}$ denote the class of starlike functions, i.e., the class of all functions $f \in \mathcal{A}$ such that

$$
\operatorname{Re} \frac{z f^{\prime}(z)}{f(z)}>0, \quad z \in \mathbb{D}
$$

Given $\delta \in(-\pi / 2, \pi / 2)$ and $g \in \mathcal{S}^{*}$, let $\mathcal{C}_{\delta}(g)$ denote the class of functions called close-toconvex with argument $\delta$ with respect to $g$, i.e., the class of all functions $f \in \mathcal{A}$ such that

$$
\operatorname{Re}\left\{\mathrm{e}^{\mathrm{i} \delta} \frac{z f^{\prime}(z)}{g(z)}\right\}>0, \quad z \in \mathbb{D} \text {. }
$$

Let

$$
\mathcal{C}(g):=\bigcup_{\delta \in(-\pi / 2, \pi / 2)} \mathcal{C}_{\delta}(g), \quad \mathcal{C}_{\delta}:=\bigcup_{g \in \mathcal{S}^{*}} \mathcal{C}_{\delta}(g)
$$

denote the classes of functions called close-to-convex with respect to $g$ and close-to-convex with argument $\delta$, respectively, and let

$$
\mathcal{C}:=\bigcup_{\delta \in(-\pi / 2, \pi / 2)} \mathcal{C}_{\delta}=\bigcup_{\delta \in(-\pi / 2, \pi / 2)} \bigcup_{g \in \mathcal{S}^{*}} \mathcal{C}_{\delta}(g)
$$

denote the class of close-to-convex functions (see [11, pp.184-185], [12,13]). It is well known that $\mathcal{S}^{*}$ and $\mathcal{C}$ are the subclasses of $\mathcal{S}$.

By using a specific starlike function $g$, inequality (1.3) defines the related class $\mathcal{C}_{\delta}(g)$. Given $\alpha \in[0,1]$, let

$$
g_{\alpha}(z):=\frac{z}{(1-\alpha z)^{2}}=\sum_{n=1}^{\infty} n \alpha^{n-1} z^{n}, \quad z \in \mathbb{D} .
$$

It is easy to check that each $g_{\alpha}$ satisfies (1.2), i.e., $g_{\alpha} \in \mathcal{S}^{*}$ for every $\alpha \in[0,1]$. Then (1.3) is of the form

$$
\operatorname{Re}\left\{\mathrm{e}^{\mathrm{i} \delta}(1-\alpha z)^{2} f^{\prime}(z)\right\}>0, \quad z \in \mathbb{D},
$$

and defines the class $\mathcal{C}_{\delta}\left(g_{\alpha}\right)$, and further the class $\mathcal{C}\left(g_{\alpha}\right)$. Such classes of functions were studied in [14,15] and [16], where some generalization of the Robertson condition for convexity in one direction [17] was discussed.

Note that for $\alpha:=1$ we get the Koebe function $g_{1}=: k$. Then condition (1.5) defines the class $\mathcal{C}_{\delta}(k)$ and further the class $\mathcal{C}(k)$ of functions close-to-convex with respect to the Koebe function. Such functions have a well-known geometrical meaning, namely condition (1.5) geometrically says that the function $h:=\mathrm{e}^{\mathrm{i} \delta} f$ has the boundary normalization

$$
\lim _{t \rightarrow \infty} h^{-1}(h(z)+t)=1
$$


and $h(\mathbb{D})$ is a domain such that $\{w+t: t \geq 0\} \subset h(\mathbb{D})$ for every $w \in h(\mathbb{D})$. Such functions $h$, clearly univalent as close-to-convex, and domains $h(\mathbb{D})$ are called convex in the positive (negative) direction of the real axis and are related to functions convex in the direction of the imaginary axis (see, e.g., [17-20], [21, Chapter VI], [22]).

For $\alpha:=0$ we have the identity function $g_{0}(z)=z, z \in \mathbb{D}$, and then condition (1.5) is of the form

$$
\operatorname{Re}\left\{\mathrm{e}^{\mathrm{i} \delta} f^{\prime}(z)\right\}>0, \quad z \in \mathbb{D} \text {. }
$$

Functions $f$ having such a property are called of bounded turning with argument $\delta$ and form the class $\mathcal{C}_{\delta}\left(g_{0}\right)$ denoted usually as $\mathcal{P}^{\prime}(\delta)$. Functions in the class $\mathcal{P}^{\prime}:=\mathcal{C}\left(g_{0}\right)$ are usually called of bounded turning ( $c f$. [23, Vol. I, p.101]). On the other hand, condition (1.6) is known as the famous criterium of univalence due to Noshiro [24] and Warschawski [25] (cf. [23, Vol. I, p.88]). In this way condition (1.5) creates a simple parametric passage from the class $\mathcal{P}^{\prime}(\delta)$ to the class $\mathcal{C}_{\delta}(k)$.

The main goal of this paper is to study the Fekete-Szegö problem for the classes $\mathcal{C}\left(g_{\alpha}\right)$, $\alpha \in[0,1]$. For the class $\mathcal{C}(k)$, i.e., for $\alpha=1$, the Fekete-Szegö problem was examined in [26], where it was shown that

$$
\max _{f \in \mathcal{C}(k)} \Phi_{\lambda}(f) \leq \begin{cases}|3-4 \lambda|, & \lambda \in(-\infty, 1 / 3] \cup[1,+\infty), \\ \frac{1}{3} \cdot \frac{(2-3 \lambda)^{2}}{2-|2-3 \lambda|}+|1-\lambda|+\frac{2}{3}, & \lambda \in[1 / 3,1],\end{cases}
$$

with sharpness of the result for $\lambda \in \mathbb{R} \backslash(2 / 3,1)$. Recall here that in [3] Keogh and Merkes proved that

$$
\max _{f \in \mathcal{C}_{0}} \Phi_{\lambda}(f)= \begin{cases}|3-4 \lambda|, & \lambda \in(-\infty, 1 / 3] \cup[1,+\infty), \\ 1 / 3+4 /(9 \lambda), & \lambda \in[1 / 3,2 / 3], \\ 1, & \lambda \in[2 / 3,1] .\end{cases}
$$

For $\lambda \in[0,1]$ Koepf in [5] extended the above result for the class of close-to-convex functions showing that

$$
\max _{f \in \mathcal{C}} \Phi_{\lambda}(f)=\max _{f \in \mathcal{C}_{0}} \Phi_{\lambda}(f)
$$

For other results on the Fekete-Szegö problem for various subclasses of close-to-convex functions, particularly for strongly close-to-convex functions, see [27-29] and [30].

For the class $\mathcal{P}^{\prime}$ and $\lambda \in[0,1]$, we get the following sharp result published, among other results, in [6, Theorem 2.3], namely

$$
\max _{f \in \mathcal{P}^{\prime}} \Phi_{\lambda}(f)=\frac{2}{3}
$$

\section{Main result}

By $\mathcal{P}$ we denote the class of all analytic functions $p$ in $\mathbb{D}$ of the form

$$
p(z)=1+\sum_{n=1}^{\infty} c_{n} z^{n}, \quad z \in \mathbb{D},
$$


having a positive real part in $\mathbb{D}$. For each $\varepsilon \in \mathbb{T}:=\{z \in \mathbb{C}:|z|=1\}$, let

$$
L_{\varepsilon}(z):=\frac{1+\varepsilon z}{1-\varepsilon z}, \quad z \in \mathbb{D}, L:=L_{1} .
$$

Inequalities (2.2) and (2.3) below are well known (see, e.g., [31, pp.41, 166]).

Lemma 2.1 If $p \in \mathcal{P}$ is of the form (2.1), then

$$
\left|c_{n}\right| \leq 2, \quad n \in \mathbb{N}
$$

and

$$
\left|c_{2}-\frac{c_{1}^{2}}{2}\right| \leq 2-\frac{\left|c_{1}\right|^{2}}{2}
$$

Both inequalities are sharp. The equality in (2.2) holds for every function $L_{\varepsilon}, \varepsilon \in \mathbb{T}$. The equality in (2.3) holds for every function

$$
\begin{aligned}
p_{t, \theta}(z) & :=t L\left(\mathrm{e}^{\mathrm{i} \theta} z\right)+(1-t) L\left(\mathrm{e}^{2 \mathrm{i} \theta} z^{2}\right) \\
& =1+2 t \mathrm{e}^{\mathrm{i} \theta} z+2 \mathrm{e}^{2 \mathrm{i} \theta} z^{2}+\cdots, \quad z \in \mathbb{D},
\end{aligned}
$$

where $t \in[0,1]$ and $\theta \in \mathbb{R}$.

Now we prove the main theorem of this paper. The source of the method of proof is in Koepf's paper [5], where the upper bound of $\Phi_{\lambda}$ for close-to-convex functions with $\lambda$ restricted to the interval $(1 / 3,2 / 3)$ was calculated. However, we modify this technique and use it homogeneously for the class $\mathcal{C}\left(g_{\alpha}\right)$ for all real $\lambda$, partially analogously as in [26] for the class $\mathcal{C}(k)$, and in [32]. We apply also the powerful Laguerre's rule of counting zeros of polynomials in an interval. We propose Laguerre's algorithm for such a computation by its simplicity, usefulness and efficiency.

We shortly recall Laguerre's rule of counting zeros of polynomials in an interval (see [33, 34], [35, pp.19-20]). Given a real polynomial

$$
Q(u)=a_{0} u^{n}+a_{1} u^{n-1}+\cdots+a_{n-1} u+a_{n},
$$

consider a finite sequence $\left(q_{k}\right), k=0,1, \ldots, n$, of polynomials of the form

$$
q_{k}(u)=\sum_{j=0}^{k} a_{j} u^{k-j} .
$$

For each $u_{0} \in \mathbb{R}$, let $N\left(Q ; u_{0}\right)$ denote the number of sign changes in the sequence $\left(q_{k}\left(u_{0}\right)\right)$, $k=0,1, \ldots, n$. Given an interval $I \subset \mathbb{R}$, denote by $Z(Q ; I)$ the number of zeros of $Q$ in $I$ counted with their orders. Then the following theorem due to Laguerre holds.

Theorem 2.2 If $a<b, Q(a) \neq 0$ and $Q(b) \neq 0$, then $Z(Q ;(a, b))=N(Q ; a)-N(Q ; b)$ or $N(Q ; a)-N(Q ; b)-Z(Q ;(a, b))$ is an even positive integer. 
Note that $q_{k}(0)=a_{k}$ and $q_{k}(1)=\sum_{j=0}^{k} a_{j}$. Thus, when $(a, b):=(0,1)$, Theorem 2.2 reduces to the following useful corollary.

Corollary 2.3 If $Q(0) \neq 0$ and $Q(1) \neq 0$, then $Z(Q ;(0,1))=N(Q ; 0)-N(Q ; 1)$ or $N(Q ; 0)-$ $N(Q ; 1)-Z(Q ;(0,1))$ is an even positive integer, where $N(Q ; 0)$ and $N(Q ; 1)$ are the numbers of sign changes in the sequence of polynomial coefficients $\left(a_{k}\right)$ and in the sequence of sums $\left(\sum_{j=0}^{k} a_{j}\right)$, with $k=0,1, \ldots, n$, respectively.

The main theorem of the paper is as follows.

Theorem 2.4 Let $\alpha \in[0,1]$. Then

$$
\begin{aligned}
& \max _{f \in \mathcal{C}\left(g_{\alpha}\right)} \Phi_{\lambda}(f) \\
& \quad \leq \begin{cases}\left|\frac{2}{3}+\frac{4}{3} \alpha+\alpha^{2}-(1+\alpha)^{2} \lambda\right|, & \lambda \in \mathbb{R} \backslash\left(\tau_{1}(\alpha), \tau_{2}(\alpha)\right), \\
\frac{2}{3}+\alpha^{2}\left(\frac{1}{3} \cdot \frac{(2-3 \lambda)^{2}}{2-|2-3 \lambda|}+|1-\lambda|\right), & \lambda \in\left[\tau_{1}(\alpha), \tau_{2}(\alpha)\right],\end{cases}
\end{aligned}
$$

where

$$
\tau_{1}(\alpha):=\frac{2 \alpha}{3(1+\alpha)}, \quad \tau_{2}(\alpha):=\frac{2(2+\alpha)}{3(1+\alpha)}
$$

For each $\alpha \in(0,1]$ and each $\lambda \in \mathbb{R} \backslash\left(2 / 3, \tau_{2}(\alpha)\right)$, as well as for $\alpha:=0$ and each $\lambda \in \mathbb{R}$, the inequality is sharp and the equality is attained by a function in $\mathcal{C}_{0}\left(g_{\alpha}\right)$. In particular,

(i) when $\alpha \in(0,1]$, for each $\lambda \in\left[\tau_{1}(\alpha), 2 / 3\right]$ the second equality in (2.7) is attained by the function $f_{\alpha, t_{\alpha, \lambda}}$ given by the differential equation

$$
f_{\alpha, t_{\alpha, \lambda}}^{\prime}(z)=\frac{p_{t_{\alpha, \lambda}, 0}(z)}{(1-\alpha z)^{2}}, \quad f_{\alpha, t_{\alpha, \lambda}}(0):=0, z \in \mathbb{D}
$$

where $t_{\alpha, \lambda}:=\alpha(2 /(3 \lambda)-1)$;

(ii) when $\alpha \in(0,1]$, for each $\lambda \in \mathbb{R} \backslash\left(\tau_{1}(\alpha), \tau_{2}(\alpha)\right)$ the first equality in (2.7) is attained by the function $f_{\alpha, 1}$, given by (2.8) with $t_{\alpha, \lambda} \equiv 1$, i.e., when $\alpha \in(0,1)$, by the function

$$
f_{\alpha, 1}(z)=\frac{2}{(1-\alpha)^{2}} \log \frac{1-\alpha z}{1-z}-\frac{1+\alpha}{1-\alpha} \cdot \frac{z}{1-\alpha z}, \quad z \in \mathbb{D}, \log 1:=0
$$

and when $\alpha=1$, by the Koebe function $f_{1,1}:=k$;

(iii) when $\alpha=0$, for each $\lambda \in[0,4 / 3]$ the second equality in (2.7) is attained by the function

$$
f_{0,0}(z):=-z+\log \frac{1+z}{1-z}, \quad z \in \mathbb{D}, \log 1:=0
$$

and for each $\lambda \in \mathbb{R} \backslash(0,4 / 3)$ the first equality in (2.7) is attained by the function

$$
f_{0,1}(z):=-z-2 \log (1-z), \quad z \in \mathbb{D}, \log 1:=0 .
$$

Proof Fix $\alpha \in[0,1]$. Observe from (1.5) that $f \in \mathcal{C}\left(g_{\alpha}\right)$ if and only if

$$
\mathrm{e}^{\mathrm{i} \delta}(1-\alpha z)^{2} f^{\prime}(z)=p(z) \cos \delta+\mathrm{i} \sin \delta, \quad z \in \mathbb{D}
$$


for some $\delta \in(-\pi / 2, \pi / 2)$ and $p \in \mathcal{P}$. Thus

$$
z f^{\prime}(z)=\mathrm{e}^{-\mathrm{i} \delta} g_{\alpha}(z)(p(z) \cos \delta+\mathrm{i} \sin \delta), \quad z \in \mathbb{D} .
$$

Setting the series (1.1), (1.4) and (2.1) into (2.13), by comparing coefficients, we get

$$
a_{2}=\frac{1}{2}\left(c_{1} \mathrm{e}^{-\mathrm{i} \delta} \cos \delta+2 \alpha\right)
$$

and

$$
a_{3}=\frac{1}{3}\left(c_{2} \mathrm{e}^{-\mathrm{i} \delta} \cos \delta+2 \alpha c_{1} \mathrm{e}^{-\mathrm{i} \delta} \cos \delta+3 \alpha^{2}\right)
$$

Let $\lambda \in \mathbb{R}$. Using (2.3), from (2.14) and (2.15), we have

$$
\begin{aligned}
\Phi_{\lambda}(f)= & \left|a_{3}-\lambda a_{2}^{2}\right| \\
= & \mid \frac{1}{3} c_{2} \mathrm{e}^{-\mathrm{i} \delta} \cos \delta+\frac{2}{3} \alpha c_{1} \mathrm{e}^{-\mathrm{i} \delta} \cos \delta+\alpha^{2} \\
& -\frac{1}{4} \lambda\left(c_{1}^{2} \mathrm{e}^{-2 \mathrm{i} \delta} \cos ^{2} \delta+4 \alpha c_{1} \mathrm{e}^{-\mathrm{i} \delta} \cos \delta+4 \alpha^{2}\right) \mid \\
= & \mid \alpha^{2}(1-\lambda)+\frac{1}{3}\left(c_{2}-\frac{c_{1}^{2}}{2}\right) \mathrm{e}^{-\mathrm{i} \delta} \cos \delta \\
& +\frac{c_{1}^{2}}{6}\left(1-\frac{3}{2} \lambda \mathrm{e}^{-\mathrm{i} \delta} \cos \delta\right) \mathrm{e}^{-\mathrm{i} \delta} \cos \delta+\alpha\left(\frac{2}{3}-\lambda\right) c_{1} \mathrm{e}^{-\mathrm{i} \delta} \cos \delta \mid \\
\leq & \alpha^{2}|1-\lambda|+\frac{1}{3}\left(2-\frac{\left|c_{1}\right|^{2}}{2}\right) \cos \delta \\
& +\frac{\left|c_{1}\right|^{2}}{6}\left|1-\frac{3}{2} \lambda \mathrm{e}^{-\mathrm{i} \delta} \cos \delta\right| \cos \delta+\alpha\left|\frac{2}{3}-\lambda\right|\left|c_{1}\right| \cos \delta \\
= & \alpha^{2}|1-\lambda| \\
& +\left(\frac{2}{3}+\frac{\left|c_{1}\right|^{2}}{6}\left(\sqrt{\left.\left.1-\left(3 \lambda-\frac{9}{4} \lambda^{2}\right) \cos ^{2} \delta-1\right)+\alpha\left|\frac{2}{3}-\lambda\right|\left|c_{1}\right|\right) \cos \delta .}\right.\right.
\end{aligned}
$$

Set $x:=\left|c_{1}\right|$ and $y:=\cos \delta$. Clearly, $y \in(0,1]$ and, in view of $(2.2), x \in[0,2]$. It is convenient to use $\gamma:=2-3 \lambda$ instead of $\lambda$ in further computation. For $\gamma \in \mathbb{R}$, let

$$
s_{\gamma}(y):=\sqrt{1-\left(1-\frac{1}{4} \gamma^{2}\right) y^{2}}, \quad y \in[0,1] .
$$

Set $R:=[0,2] \times[0,1]$. For $\alpha \in[0,1]$ and $\gamma \in \mathbb{R}$, define

$$
F_{\alpha, \gamma}(x, y):=\frac{1}{3} \alpha^{2}|1+\gamma|+\frac{1}{3}\left(2+\frac{x^{2}}{2}\left(s_{\gamma}(y)-1\right)+\alpha|\gamma| x\right) y, \quad(x, y) \in R
$$

Consequently, in view of (2.16) we have

$$
\max _{f \in \mathcal{C}\left(g_{\alpha}\right)} \Phi_{\lambda}(f) \leq \max _{(x, y) \in R} F_{\alpha, \gamma}(x, y)
$$


Now, for each $\alpha \in[0,1]$ and $\gamma \in \mathbb{R}$, we find the maximum value of $F_{\alpha, \gamma}$ on the rectangle $R$.

1. In the corners of $R$ we have

$$
\begin{aligned}
& F_{\alpha, \gamma}(0,0)=F_{\alpha, \gamma}(2,0)=\frac{1}{3} \alpha^{2}|1+\gamma|, \\
& F_{\alpha, \gamma}(0,1)=\frac{1}{3} \alpha^{2}|1+\gamma|+\frac{2}{3}, \\
& F_{\alpha, \gamma}(2,1)=\frac{1}{3} \alpha^{2}|1+\gamma|+\frac{1}{3}(1+2 \alpha)|\gamma| .
\end{aligned}
$$

2. For $x=0$ and $y \in(0,1)$ we have a linear function and for $x \in(0,2)$ and $y=0$ we have a constant function.

3. For $x \in(0,2)$ and $y=1$, let

$$
G_{\alpha, \gamma}(x):=F_{\alpha, \gamma}(x, 1)=\frac{1}{3}\left(\frac{1}{4}(|\gamma|-2) x^{2}+\alpha|\gamma| x+\alpha^{2}|1+\gamma|+2\right) .
$$

For $|\gamma|=2$ we get the linear functions, so let $|\gamma| \neq 2$. Then $G_{\alpha, \gamma}^{\prime}(x)=0$ if and only if

$$
x=\frac{2 \alpha|\gamma|}{2-|\gamma|}=: x_{\alpha, \gamma}
$$

Thus $x_{\alpha, \gamma} \in(0,2)$ if and only if

$$
\alpha \neq 0 \wedge 0<|\gamma|<\frac{2}{1+\alpha} .
$$

Moreover, we have

$$
F_{\alpha, \gamma}\left(x_{\alpha, \gamma}, 1\right)=\frac{1}{3}\left(\frac{\alpha^{2} \gamma^{2}}{2-|\gamma|}+\alpha^{2}|1+\gamma|+2\right) \text {. }
$$

4. For $x=2$ and $y \in(0,1)$, let

$$
H_{\alpha, \gamma}(y):=F_{\alpha, \gamma}(2, y)=\frac{1}{3}\left(\alpha^{2}|1+\gamma|+2 y s_{\gamma}(y)+2 \alpha|\gamma| y\right) .
$$

For $|\gamma|=2$ we have the linear functions, evidently, so let $|\gamma| \neq 2$. Note first that

$$
s_{\gamma}(y)>0, \quad y \in(0,1) \text {. }
$$

Taking into account (2.22), we have

$$
y s_{\gamma}^{\prime}(y)=\frac{-\left(1-\frac{1}{4} \gamma^{2}\right) y^{2}}{\sqrt{1-\left(1-\frac{1}{4} \gamma^{2}\right) y^{2}}}=\frac{s_{\gamma}^{2}(y)-1}{s_{\gamma}(y)}, \quad y \in(0,1) .
$$

Using (2.23) we get

$$
H_{\alpha, \gamma}^{\prime}(y)=\frac{2}{3}\left(s_{\gamma}(y)+\frac{s_{\gamma}^{2}(y)-1}{s_{\gamma}(y)}+\alpha|\gamma|\right)=0, \quad y \in(0,1)
$$


if and only if

$$
2 s_{\gamma}^{2}(y)+\alpha|\gamma| s_{\gamma}(y)-1=0, \quad y \in(0,1)
$$

i.e., in view of (2.22) if and only if

$$
s_{\gamma}(y)=\frac{-\alpha|\gamma|+\sqrt{8+\alpha^{2} \gamma^{2}}}{4}=: s_{\alpha, \gamma}, \quad y \in(0,1) \text {. }
$$

Since $|\gamma| \neq 2$, so from the above we get the equation

$$
y^{2}=\frac{4-\alpha^{2} \gamma^{2}+\alpha|\gamma| \sqrt{8+\alpha^{2} \gamma^{2}}}{2\left(4-\gamma^{2}\right)}, \quad y \in(0,1) .
$$

Thus the solution of equation (2.25), and hence of (2.24), exists if and only if

$$
0<\frac{4-\alpha^{2} \gamma^{2}+\alpha|\gamma| \sqrt{8+\alpha^{2} \gamma^{2}}}{2\left(4-\gamma^{2}\right)}<1 .
$$

Elementary computing shows that (2.26) holds if and only if

$$
|\gamma|<\sqrt{\frac{2}{1+\alpha}}
$$

Thus the function $H_{\alpha, \gamma}$ has a critical point in $(0,1)$, namely

$$
y=\sqrt{\frac{4-\alpha^{2} \gamma^{2}+\alpha|\gamma| \sqrt{\alpha^{2} \gamma^{2}+8}}{2\left(4-\gamma^{2}\right)}}=: y_{\alpha, \gamma},
$$

as the unique solution of (2.25), if and only if (2.27) holds. Moreover,

$$
\begin{aligned}
F_{\alpha, \gamma}\left(2, y_{\alpha, \gamma}\right)= & \frac{1}{3} \alpha^{2}|1+\gamma| \\
& +\frac{1}{6} \sqrt{\frac{4-\alpha^{2} \gamma^{2}+\alpha|\gamma| \sqrt{8+\alpha^{2} \gamma^{2}}}{2\left(4-\gamma^{2}\right)}}\left(\sqrt{8+\alpha^{2} \gamma^{2}}+3 \alpha|\gamma|\right) .
\end{aligned}
$$

5. We will prove that for each $\alpha \in[0,1]$ and $\gamma \in \mathbb{R}$ the function $F_{\alpha, \gamma}$ has no critical point in $(0,2) \times(0,1)$.

Since $y \neq 0$ and $x \neq 0$, we have

$$
\frac{\partial F_{\alpha, \gamma}}{\partial x}=0
$$

if and only if

$$
s_{\gamma}(y)=1-\frac{\alpha|\gamma|}{x}, \quad y \in(0,1)
$$

Since $x>0$, by comparing (2.29) and (2.22), we see that $x>\alpha|\gamma|$. By a simple observation, we deduce that the solution of (2.29) can exist only when

$$
(\alpha=0 \wedge|\gamma|=2) \vee(\alpha \neq 0 \wedge \gamma \neq 0 \wedge|\gamma| \neq 2 \wedge x>\alpha|\gamma|) .
$$


Squaring then (2.29), we obtain

$$
s_{\gamma}^{2}(y)-1=-\frac{2 \alpha|\gamma|}{x}+\frac{\alpha^{2} \gamma^{2}}{x^{2}} .
$$

Since by (2.22), $s_{\gamma}(y) \neq 0$ for $y \in(0,1)$, taking into account (2.23), we have

$$
\frac{\partial F_{\alpha, \gamma}}{\partial y}=\frac{2}{3}+\frac{x^{2}}{6}\left(s_{\gamma}(y)-1\right)+\frac{1}{3} \alpha|\gamma| x+\frac{x^{2}}{6} \cdot \frac{s_{\gamma}^{2}(y)-1}{s_{\gamma}(y)} .
$$

Thus, by using (2.29) and (2.31), after simplifying we have

$$
\frac{\partial F_{\alpha, \gamma}}{\partial y}=0
$$

if and only if

$$
\alpha|\gamma| x^{2}-4 x+4 \alpha|\gamma|=0, \quad x \in(0,2)
$$

It follows at once that for $\alpha=0$ and $\gamma \in \mathbb{R}$, as well as for $\alpha \in(0,1]$ and $|\gamma| \geq 1 / \alpha$, equation (2.32) has no root. Thus by (2.30) we consider

$$
\alpha \neq 0 \wedge|\gamma| \neq 2 \wedge 0<|\gamma|<1 / \alpha \wedge x>\alpha|\gamma|
$$

Solving now (2.32), we have

$$
x=\frac{2-2 \sqrt{1-\alpha^{2} \gamma^{2}}}{\alpha|\gamma|}=: x_{1 ; \alpha, \gamma}, \quad x=\frac{2+2 \sqrt{1-\alpha^{2} \gamma^{2}}}{\alpha|\gamma|}=: x_{2 ; \alpha, \gamma} .
$$

Clearly, $x_{2 ; \alpha, \gamma} \notin(0,2)$ and it remains to consider $x_{1 ; \alpha, \gamma}$. It is easy to check that $x_{1 ; \alpha, \gamma}>\alpha|\gamma|$. Thus setting $x:=x_{1 ; \alpha, \gamma}$ into (2.31) and computing, we have

$$
y^{2}=\frac{\frac{2 \alpha|\gamma|}{x_{1 ; \alpha, \gamma}}-\frac{\alpha^{2} \gamma^{2}}{x_{1 ; \alpha, \gamma}^{2}}}{1-\frac{1}{4} \gamma^{2}}=\frac{\left(4-\alpha^{2} \gamma^{2}-4 \sqrt{1-\alpha^{2} \gamma^{2}}\right) \alpha^{2} \gamma^{2}}{\left(1-\sqrt{1-\alpha^{2} \gamma^{2}}\right)^{2}\left(4-\gamma^{2}\right)} .
$$

A solution in $(0,1)$ of $(2.34)$ exists if and only if

$$
0<\frac{\left(4-\alpha^{2} \gamma^{2}-4 \sqrt{1-\alpha^{2} \gamma^{2}}\right) \alpha^{2} \gamma^{2}}{\left(1-\sqrt{1-\alpha^{2} \gamma^{2}}\right)^{2}\left(4-\gamma^{2}\right)}<1 .
$$

By (2.33) consider

$$
\alpha \neq 0 \wedge|\gamma| \neq 2 \wedge 0<|\gamma|<\frac{1}{\alpha} .
$$

We prove that then condition (2.35) is false. When $2<|\gamma|<1 / \alpha$, then an easy computation shows that the left-hand inequality in (2.35) is false. Thus by (2.36) it remains to consider

$$
\alpha \neq 0 \wedge 0<|\gamma|<\frac{1}{\alpha} \leq 2
$$


By an easy computation we check that then the left-hand inequality in (2.35) holds. Since $4-\gamma^{2}>0$, write the right-hand inequality in (2.35) as

$$
\left(8-2\left(1+2 \alpha^{2}\right) \gamma^{2}\right) \sqrt{1-\alpha^{2} \gamma^{2}}<\left(\alpha^{4}+\alpha^{2}\right) \gamma^{4}-2\left(1+4 \alpha^{2}\right) \gamma^{2}+8 .
$$

The last step is to show, which does not cause difficulties, that under the assumption (2.37) the above inequality is false. We omit the details.

Summarizing, we proved that condition (2.35) is false, so equation (2.34) has no solution in $(0,1)$.

Thus the proof that for $\alpha \in[0,1]$ and $\gamma \in \mathbb{R}$ the function $F_{\alpha, \gamma}$ has no critical point in $(0,2) \times(0,1)$ is finished.

6. Now we calculate the maximum value of $F_{\alpha, \gamma}$ in $R$, which, as was shown, is attained on the boundary of $R$. Let $\alpha \in[0,1]$. Taking into account Part 3 with (2.20) and Part 4 with (2.27), we consider the following cases.

(A) $|\gamma| \geq 2 /(1+\alpha)$. Then the maximum value of $F_{\alpha, \gamma}$ is attained in a corner of $R$. Thus by (2.17)-(2.19) an easy computation shows that

$$
\max _{(x, y) \in R} F_{\alpha, \gamma}(x, y)=F_{\alpha, \gamma}(2,1)=\frac{1}{3}\left|\alpha^{2}+(1+\alpha)^{2} \gamma\right| \text {. }
$$

(B) $\sqrt{2 /(1+\alpha)} \leq|\gamma|<2 /(\alpha+1)$. Then the maximum value of $F_{\alpha, \gamma}$ is attained in a corner of $R$ or in $\left(x_{\alpha, \gamma}, 1\right)$. Thus, by (2.17)-(2.19) and (2.21), we calculate that

$$
\max _{(x, y) \in R} F_{\alpha, \gamma}(x, y)=F_{\alpha, \gamma}\left(x_{\alpha, \gamma}, 1\right)=\frac{1}{3} \cdot \frac{\alpha^{2} \gamma^{2}}{2-|\gamma|}+\frac{1}{3} \alpha^{2}|1+\gamma|+\frac{2}{3} .
$$

(C) $\gamma=0$. Then the maximum value of $F_{\alpha, 0}$ is attained in a corner of $R$ or in the point $\left(2, y_{\alpha, 0}\right)=(2,1 / \sqrt{2})$. Thus, by $(2.17)-(2.19)$ and $(2.28)$ with $\gamma:=0$, we see that

$$
\max _{(x, y) \in R} F_{\alpha, 0}(x, y)=F_{\alpha, 0}(0,1)=\frac{1}{3} \alpha^{2}+\frac{2}{3} .
$$

(D) $0<|\gamma|<\sqrt{2 /(\alpha+1)}$. Then we compare all values (2.17)-(2.19), and by (2.21) and (2.28), $F_{\alpha, \gamma}\left(x_{\alpha, \gamma}, 1\right)$ and $F_{\alpha, \gamma}\left(2, y_{\alpha, \gamma}\right)$. We will show that the value $F_{\alpha, \gamma}\left(x_{\alpha, \gamma}, 1\right)$ is the largest one. As it is easy to observe, it is enough to prove that

$$
F_{\alpha, \gamma}\left(x_{\alpha, \gamma}, 1\right) \geq F_{\alpha, \gamma}\left(2, y_{\alpha, \gamma}\right)
$$

i.e., in view of (2.21) and (2.28), after a simple computation, we have

$$
2\left(2+\frac{\alpha^{2} \gamma^{2}}{2-|\gamma|}\right) \geq \sqrt{\frac{4-\alpha^{2} \gamma^{2}+\alpha|\gamma| \sqrt{8+\alpha^{2} \gamma^{2}}}{2\left(4-\gamma^{2}\right)}}\left(\sqrt{8+\alpha^{2} \gamma^{2}}+3 \alpha|\gamma|\right) .
$$

As $|\gamma|<2$, so squaring (2.42) and computing, we equivalently have

$$
\begin{aligned}
& \alpha^{4}|\gamma|^{5}+\left(6 \alpha^{4}-8 \alpha^{2}\right) \gamma^{4}+\left(20 \alpha^{2}+8\right)|\gamma|^{3}-\left(8 \alpha^{2}+16\right) \gamma^{2}-24|\gamma|+48 \\
& \quad \geq \alpha|\gamma|(2-|\gamma|)\left(\alpha^{2} \gamma^{2}+8\right)^{3 / 2} .
\end{aligned}
$$


To verify that (2.43) holds, setting $u_{\alpha}:=\sqrt{2 /(\alpha+1)}$, we will show that for every $\alpha \in[0,1]$ we have

$$
\begin{aligned}
Q_{\alpha}(u) & :=\alpha^{4} u^{5}+\left(6 \alpha^{4}-8 \alpha^{2}\right) u^{4}+\left(20 \alpha^{2}+8\right) u^{3}-\left(8 \alpha^{2}+16\right) u^{2}-24 u+48 \\
& \geq \alpha u(2-u)\left(\alpha^{2} u^{2}+8\right)^{3 / 2}=: S_{\alpha}(u), \quad u \in\left[0, u_{\alpha}\right] .
\end{aligned}
$$

$\left(1^{\circ}\right)$ For $\alpha=0$, inequality (2.43) is evidently true.

$\left(2^{\circ}\right)$ For $\alpha=1$, we have $u_{1}=1$ and inequality (2.44) after computing is equivalent to the evidently true inequality

$$
(u-1)^{2}\left(2 u^{6}+32 u^{4}+40 u^{3}-92 u^{2}+144 u+144\right) \geq 0, \quad u \in[0,1] .
$$

$\left(^{\circ}\right)$ Let $\alpha \in(0,1)$. We will show that for $u \in\left[0, u_{\alpha}\right]$,

$$
V_{\alpha}(u):=Q_{\alpha}^{2}(u)-S_{\alpha}^{2}(u)=\left(Q_{\alpha}(u)-S_{\alpha}(u)\right)\left(Q_{\alpha}(u)+S_{\alpha}(u)\right)>0 .
$$

Further, taking into account that $Q_{\alpha}$ and $S_{\alpha}$ are continuous functions with

$$
Q_{\alpha}(0)-S_{\alpha}(0)=48>0,
$$

from (2.45) we deduce that

$$
Q_{\alpha}(u)-S_{\alpha}(u)>0, \quad u \in\left[0, u_{\alpha}\right]
$$

which confirms (2.44) and further (2.43).

Now we prove that (2.45) holds, i.e., after computation we have

$$
\begin{aligned}
V_{\alpha}(u)= & \left(\alpha^{8}-\alpha^{6}\right) u^{9}+\left(2 \alpha^{8}-5 \alpha^{6}+5 \alpha^{4}\right) u^{8} \\
& +\left(20 \alpha^{6}-16 \alpha^{4}-8 \alpha^{2}\right) u^{7}+\left(-12 \alpha^{6}+6 \alpha^{4}+36 \alpha^{2}+4\right) u^{6} \\
& +\left(16 \alpha^{4}-24 \alpha^{2}-16\right) u^{5}-\left(8 \alpha^{4}+124 \alpha^{2}+8\right) u^{4} \\
& +\left(272 \alpha^{2}+96\right) u^{3}-\left(176 \alpha^{2}+60\right) u^{2}-144 u+144 \\
> & 0, \quad u \in\left[0, u_{\alpha}\right] .
\end{aligned}
$$

As in (2.6), let $\left(q_{k}\right), k=0,1, \ldots, 9$, be a sequence of polynomials of the form

$$
q_{k}(u)=\sum_{j=0}^{k} a_{j} u^{k-j}, \quad u \in\left[0, u_{\alpha}\right],
$$

corresponding to the polynomial $Q:=V_{\alpha}$ in (2.5) for Laguerre's rule in $\left[0, u_{\alpha}\right]$.

(a) First we check the signs of the elements of the sequence $\left(q_{k}(0)\right)$, i.e., of the sequence $\left(a_{k}\right)$ for $k=0,1, \ldots, 9$. A simple computation shows that for $\alpha \in(0,1)$ we have $q_{0}(0)<0$, $q_{1}(0)>0, q_{2}(0)<0, q_{3}(0)>0, q_{4}(0)<0, q_{5}(0)<0, q_{6}(0)>0, q_{7}(0)<0, q_{8}(0)<0$ and $q_{9}(0)>0$. Hence

$$
N\left(V_{\alpha} ; 0\right)=7, \quad \alpha \in(0,1) .
$$


(b) Now we check the signs of the elements of the sequence $\left(q_{k}\left(u_{\alpha}\right)\right)$ for $k=0,1, \ldots, 9$. After the detailed computation and arguments based on Laguerre's rule, we show that $q_{0}\left(u_{\alpha}\right)<0, q_{1}\left(u_{\alpha}\right)>0, q_{2}\left(u_{\alpha}\right)<0, q_{3}\left(u_{\alpha}\right)>0, q_{5}\left(u_{\alpha}\right)<0, q_{6}\left(u_{\alpha}\right)>0, q_{7}\left(u_{\alpha}\right)>0, q_{8}\left(u_{\alpha}\right)<0$ and $q_{9}\left(u_{\alpha}\right)>0$. Moreover, we show that there exists a unique $\alpha_{0} \in(0,1)$ such that $q_{4}\left(u_{\alpha_{0}}\right)=$ 0 and $q_{4}\left(u_{\alpha}\right)<0$ for $\alpha \in\left(0, \alpha_{0}\right)$ and $q_{4}\left(u_{\alpha}\right)>0$ for $\alpha \in\left(\alpha_{0}, 1\right)$. Thus for three cases, namely for $\alpha \in\left(0, \alpha_{0}\right), \alpha:=\alpha_{0}$ and $\alpha \in\left(\alpha_{0}, 1\right)$, we have

$$
N\left(V_{\alpha} ; u_{\alpha}\right)=7 .
$$

Hence, by (2.46) and by Corollary 2.3, we conclude that for each $\alpha \in(0,1)$ the polynomial $V_{\alpha}$ has no zero in $\left(0, u_{\alpha}\right)$, and since $V_{\alpha}(0)=144>0$, so $(2.45)$ holds.

Now we shortly explain the method of describing the signs of $q_{k}\left(u_{\alpha}\right), k=1, \ldots, 9$. Note that the case $k=0$ is evident since

$$
q_{0}\left(u_{\alpha}\right)=\alpha^{6}\left(\alpha^{2}-1\right)<0, \quad \alpha \in(0,1) .
$$

For other cases, i.e., for $k=1, \ldots, 9$, we use Laguerre's rule in each case in the same manner. We clarify this for the case $k=2$. We have

$$
q_{2}(u)=\left(\alpha^{8}-\alpha^{6}\right) u^{2}+\left(2 \alpha^{8}-5 \alpha^{6}+5 \alpha^{4}\right) u+20 \alpha^{6}-16 \alpha^{4}-8 \alpha^{2}, \quad u \in\left[0, u_{\alpha}\right] .
$$

We will show that

$$
q_{2}\left(u_{\alpha}\right)<0, \quad \alpha \in(0,1),
$$

i.e., after computing we get

$$
\sqrt{2} \alpha^{2}\left(2 \alpha^{4}-5 \alpha^{2}+5\right)<\left(-2 \alpha^{5}-18 \alpha^{4}+16 \alpha^{2}+8\right) \sqrt{\alpha+1}, \quad \alpha \in(0,1) .
$$

To verify that (2.48) holds, we will show that

$$
\begin{aligned}
r(t) & :=\sqrt{2} t^{2}\left(2 t^{4}-5 t^{2}+5\right) \\
& <\left(-2 t^{5}-18 t^{4}+16 t^{2}+8\right) \sqrt{t+1}=: s(t), \quad t \in[0,1] .
\end{aligned}
$$

Applying Corollary 2.3, we see that

$$
\begin{aligned}
w(t):= & s^{2}(t)-r^{2}(t) \\
= & -8 t^{12}+4 t^{11}+116 t^{10}+396 t^{9}+170 t^{8}-640 t^{7}-508 t^{6} \\
& -64 t^{5}-82 t^{4}+256 t^{3}+256 t^{2}+64 t+64 \\
= & \sum_{j=0}^{12} b_{j} t^{12-j}>0, \quad t \in[0,1] .
\end{aligned}
$$

Indeed, the numbers of sign changes in the sequence of polynomial coefficients $\left(b_{k}\right)$ and in the sequence of sums $\left(\sum_{j=0}^{k} b_{j}\right)$, where $k=0,1, \ldots, 12$, equal 3, i.e., $N(w ; 0)=N(w ; 1)=3$. Thus we conclude that the polynomial $w$ has no zero in the interval $(0,1)$ and, since $w(0)=$ 
$64>0$, so (2.50) holds. Hence, and by the fact that $s(0)>r(0)$, we deduce that (2.49) holds, which confirms (2.47).

Note here that for the case $k=4$ we show that the equation

$$
q_{4}\left(u_{\alpha}\right)=0
$$

has a unique solution $\alpha=: \alpha_{0} \in(0,1)$. In this case, we show by using Laguerre's rule that the corresponding polynomial $w$ as in $(2.50)$ has a unique zero in $(0,1)$ and further we deduce that

$$
q_{4}\left(u_{\alpha}\right)<0, \quad \alpha \in\left(0, \alpha_{0}\right)
$$

and

$$
q_{4}\left(u_{\alpha}\right)>0, \quad \alpha \in\left(\alpha_{0}, 1\right) .
$$

Summarizing, taking into account (2.38)-(2.41), we have

$$
\max _{(x, y) \in R} F_{\alpha, \gamma}(x, y)= \begin{cases}\frac{1}{3}\left|\alpha^{2}+(1+\alpha)^{2} \gamma\right|, & |\gamma| \geq \frac{2}{1+\alpha} \\ \frac{1}{3} \cdot \frac{\alpha^{2} \gamma^{2}}{2-|\gamma|}+\frac{1}{3} \alpha^{2}|1+\gamma|+\frac{2}{3}, & |\gamma| \leq \frac{2}{1+\alpha} .\end{cases}
$$

Finally, substituting $\gamma=2-3 \lambda$, the above yields (2.7).

Now we deal with the sharpness of the result. Let $\alpha \in(0,1]$. We prove that for each $\lambda \in(-\infty, 2 / 3] \cup\left[\tau_{2}(\alpha),+\infty\right)$ inequality (2.7) is sharp. Let $\lambda \in\left[\tau_{1}(\alpha), 2 / 3\right]$. Since then

$$
\alpha^{2}\left(\frac{1}{3} \cdot \frac{(2-3 \lambda)^{2}}{2-|2-3 \lambda|}+|1-\lambda|\right)+\frac{2}{3}=\alpha^{2}\left(\frac{4}{9 \lambda}-\frac{1}{3}\right)+\frac{2}{3},
$$

inequality (2.7) is of the form

$$
\max _{f \in \mathcal{C}\left(g_{\alpha}\right)} \Phi_{\lambda}(f) \leq \alpha^{2}\left(\frac{4}{9 \lambda}-\frac{1}{3}\right)+\frac{2}{3}, \quad \lambda \in\left[\tau_{1}(\alpha), 2 / 3\right] .
$$

Let $t_{\alpha, \lambda}:=\alpha(2 /(3 \lambda)-1)$. Then $t_{\alpha, \lambda} \in[0,1]$ and, in view of $(2.4), p_{t_{\alpha, \lambda}, 0} \in \mathcal{P}$, with $c_{1}=2 t_{\alpha, \lambda}$ and $c_{2}=2$. Setting $\delta:=0$ and $p:=p_{t_{\alpha, \lambda}, 0}$ into (2.12), we get the function $f_{\alpha, t_{\alpha, \lambda}}$ given by equation (2.8) for which, by (2.14) and (2.15),

$$
a_{2}=t_{\alpha, \lambda}+\alpha=\frac{2 \alpha}{3 \lambda}, \quad a_{3}=\frac{1}{3}\left(2+4 \alpha t_{\alpha, \lambda}+3 \alpha^{2}\right)=\frac{2}{3}+\alpha^{2}\left(\frac{8}{9 \lambda}-\frac{1}{3}\right) .
$$

Hence

$$
\Phi_{\lambda}\left(f_{\alpha, t_{\alpha, \lambda}}\right)=\frac{2}{3}+\alpha^{2}\left(\frac{4}{9 \lambda}-\frac{1}{3}\right)
$$

which makes the equality in (2.51), so in (2.7). Clearly, $f_{\alpha, t_{\alpha, \lambda}} \in \mathcal{C}_{0}\left(g_{\alpha}\right)$ because (2.12) is satisfied for $\delta=0$. 
Let $\lambda \in\left(-\infty, \tau_{1}(\alpha)\right] \cup\left[\tau_{2}(\alpha),+\infty\right)$. Then inequality (2.7) is of the form

$$
\max _{f \in \mathcal{C}\left(g_{\alpha}\right)} \Phi_{\lambda}(f) \leq\left|\frac{2}{3}+\frac{4}{3} \alpha+\alpha^{2}-(1+\alpha)^{2} \lambda\right|
$$

Set $\delta:=0$ and $p:=L$ into (2.12). Then for $\alpha \in(0,1)$ we get the function $f_{\alpha, 1}$ given by (2.9) and for $\alpha=1$ we get the Koebe function $f_{1,1}=k$, with

$$
a_{2}=1+\alpha, \quad a_{3}=\frac{1}{3}\left(2+4 \alpha+3 \alpha^{2}\right), \quad \alpha \in(0,1]
$$

Hence

$$
\Phi_{\lambda}\left(f_{\alpha, 1}\right)=\left|\frac{2}{3}+\frac{4}{3} \alpha+\alpha^{2}-(1+\alpha)^{2} \lambda\right|
$$

which makes the equality in (2.52), so in (2.7). Clearly, $f_{\alpha, 1} \in \mathcal{C}_{0}\left(g_{\alpha}\right)$ for $\alpha \in(0,1]$.

Let $\alpha:=0$. We prove that for each $\lambda \in \mathbb{R}$ inequality (2.7) is sharp. For $\lambda \in\left[\tau_{1}(0), \tau_{2}(0)\right]=$ $[0,4 / 3]$ inequality $(2.7)$ is of the form

$$
\max _{f \in \mathcal{P}^{\prime}} \Phi_{\lambda}(f) \leq \frac{2}{3}
$$

Setting $\delta:=0$ and, by (2.4), $p:=p_{0,0}$ into (2.12), we get the function $f_{0,0}$ given by (2.10) with $a_{2}=0$ and $a_{3}=2 / 3$. Hence

$$
\Phi_{\lambda}\left(f_{0,0}\right)=\frac{2}{3}
$$

which makes the equality in (2.53), so in (2.7).

For $\lambda \in(-\infty, 0] \cup[4 / 3,+\infty)$ inequality $(2.7)$ is of the form

$$
\max _{f \in \mathcal{P}^{\prime}} \Phi_{\lambda}(f) \leq\left|\frac{2}{3}-\lambda\right|
$$

Setting $\delta:=0$ and $p:=L$ into (2.12), we get the function $f_{0,1}$ given by (2.11) with $a_{2}=1$ and $a_{3}=2 / 3$. Hence

$$
\Phi_{\lambda}\left(f_{0,1}\right)=\left|\frac{2}{3}-\lambda\right|
$$

which makes the equality in (2.54), so in (2.7).

Remark 2.5 Particularly, from (2.7) for $\alpha \in[0,1]$ we have

$$
\max _{f \in \mathcal{C}\left(g_{\alpha}\right)} \Phi_{\lambda}(f)= \begin{cases}\left|\frac{2}{3}+\frac{4}{3} \alpha+\alpha^{2}-(1+\alpha)^{2} \lambda\right|, & \lambda \in \mathbb{R} \backslash\left(\tau_{1}(\alpha), \tau_{2}(\alpha)\right), \\ \frac{2}{3}+\alpha^{2}\left(\frac{4}{9 \lambda}-\frac{1}{3}\right), & \lambda \in\left[\tau_{1}(\alpha), 2 / 3\right] .\end{cases}
$$

For $\alpha:=1$ we have $\tau_{1}(1)=1 / 3$ and $\tau_{2}(1)=1, g_{1}=k$, and then Theorem 2.4 reduces to the result of [26] as follows. 


\section{Corollary 2.6}

$$
\max _{f \in \mathcal{C}(k)} \Phi_{\lambda}(f) \leq \begin{cases}|3-4 \lambda|, & \lambda \in(-\infty, 1 / 3] \cup[1,+\infty) \\ \frac{2}{3}+\frac{1}{3} \cdot \frac{(2-3 \lambda)^{2}}{2-|2-3 \lambda|}+|1-\lambda|, & \lambda \in[1 / 3,1]\end{cases}
$$

For each $\lambda \in(-\infty, 2 / 3] \cup[1,+\infty)$, the inequality is sharp and the equality is attained by a function in $\mathcal{C}_{0}(k)$. In particular, for each $\lambda \in[1 / 3,2 / 3]$ the second equality in (2.55) is attained by the function $f_{t_{\lambda}}:=f_{1, t_{1, \lambda}}$ given by differential equation (2.8), where $t_{\lambda}:=t_{1, \lambda}$. For each $\lambda \in(-\infty, 1 / 3] \cup[1,+\infty)$, the first equality in (2.55) is attained by the Koebe function $f_{1}:=k$.

For $\alpha:=0$ we have $\tau_{1}(0)=0$ and $\tau_{2}(0)=4 / 3$, and Theorem 2.4 yields the following.

\section{Corollary 2.7}

$$
\max _{f \in \mathcal{P}^{\prime}} \Phi_{\lambda}(f)= \begin{cases}\left|\frac{2}{3}-\lambda\right|, & \lambda \in(-\infty, 0] \cup[4 / 3,+\infty), \\ \frac{2}{3}, & \lambda \in[0,4 / 3]\end{cases}
$$

For each $\lambda \in[0,4 / 3]$ the second equality in (2.56) is attained by the function given by (2.10). For each $\lambda \in(-\infty, 0] \cup[4 / 3,+\infty)$, the first equality in (2.56) is attained by function (2.11).

\section{Competing interests}

The authors declare that they have no competing interests.

\section{Authors' contributions}

All authors read and approved the final manuscript.

\section{Author details}

'Department of Applied Mathematics, University of Warmia and Mazury, Słoneczna 54, Olsztyn, 10-710, Poland.

${ }^{2}$ Department of Analysis and Differential Equations, University of Warmia and Mazury, Słoneczna 54, Olsztyn, 10-710, Poland.

Received: 9 December 2013 Accepted: 31 January 2014 Published: 13 Feb 2014

\section{References}

1. Fekete, M, Szegö, G: Eine Bemerkung über ungerade schlichte Funktionen. J. Lond. Math. Soc. 8, $85-89$ (1933)

2. Jakubowski, ZJ: Sur le maximum de la fonctionnelle $\left|A_{3}-\alpha A_{2}^{2}\right|(0 \leq \alpha<1)$ dans la famille de fonctions $F_{M}$. Bull. Soc. Sci. Lett. Lódź 13(1), 19 (1962)

3. Keogh, FR, Merkes, EP: A coefficient inequality for certain classes of analytic functions. Proc. Am. Math. Soc. 20, 8-12 (1969)

4. Pfluger, A: The Fekete-Szegö inequality for complex parameter. Complex Var. Theory Appl. 7, 149-160 (1986)

5. Koepf, W: On the Fekete-Szegö problem for close-to-convex functions. Proc. Am. Math. Soc. 101, 89-95 (1987)

6. Kanas, S, Lecko, A: On the Fekete-Szegö problem and the domain of convexity for a certain class of univalent functions. Folia Sci. Univ. Tech. Resov. 73, 49-57 (1990)

7. London, RR: Fekete-Szegö inequalities for close-to-convex functions. Proc. Am. Math. Soc. 117(4), $947-950$ (1993)

8. Kim, YC, Choi, JH, Sugawa, T: Coefficient bounds and convolution properties for certain classes of close-to-convex functions. Proc. Jpn. Acad. 76, 95-98 (2000)

9. Bhowmik, B, Ponnusamy, S, Wirths, KJ: On the Fekete-Szegö problem for concave univalent functions. J. Math. Anal. Appl. 373, 432-438 (2011)

10. Kanas, S: An unified approach to the Fekete-Szegö problem. Appl. Math. Comput. 218, 8453-8461 (2012)

11. Ozaki, S: On the theory of multivalent functions. Sci. Rep. Tokyo Bunrika Daigaku, Sect. A. Math. Phys. Chem. 2 167-188 (1935)

12. Kaplan, W: Close to convex schlicht functions. Mich. Math. J. 1, 169-185 (1952)

13. Goodman, AW, Saff, EB: On the definition of close-to-convex function. Int. J. Math. Math. Sci. 1, 125-132 (1978)

14. Lecko, A: Some generalization of analytic condition for class of functions convex in a given direction. Folia Sci. Univ. Tech. Resov. 121(14), 23-34 (1993) 
15. Lecko, A: A generalization of analytic condition for convexity in one direction. Demonstr. Math. XXX(1), 155-170 (1997)

16. Lecko, A, Yaguchi, T: A generalization of the condition due to Robertson. Math. Jpn. 47(1), 133-141 (1998)

17. Robertson, MS: Analytic functions star-like in one direction. Am. J. Math. 58, 465-472 (1936)

18. Hengartner, W, Schober, G: On schlicht mappings to domains convex in one direction. Comment. Math. Helv. 45, 303-314 (1970)

19. Ciozda, W: Sur la classe des fonctions convexes vers l'axe réel négatif. Bull. Acad. Pol. Sci., Sér. Sci. Math. 27(3-4), 255-261 (1979)

20. Lecko, A: On the class of functions convex in the negative direction of the imaginary axis. J. Aust. Math. Soc. 73, 1-10 (2002)

21. Lecko, A: Some Methods in the Theory of Univalent Functions. Oficyna Wydawnicza Politechniki Rzeszowskiej, Rzeszów (2005)

22. Elin, M, Khavinson, D, Reich, S, Shoikhet, D: Linearization models for parabolic dynamical systems via Abel's functional equation. Ann. Acad. Sci. Fenn., Math. 35, 439-472 (2010)

23. Goodman, AW: Univalent Functions. Mariner, Tampa (1983)

24. Noshiro, K: On the theory of schlicht functions. J. Fac. Sci., Hokkaido Univ., Ser. 1 2, 129-155 (1934-1935)

25. Warschawski, SE: On the higher derivatives at the boundary in conformal mapping. Trans. Am. Math. Soc. 38(2), 310-340 (1935)

26. Kowalczyk, B, Lecko, A: Fekete-Szegö problem for close-to-convex functions with respect to the Koebe function (submitted)

27. Koepf, W: On the Fekete-Szegö problem for close-to-convex functions II. Arch. Math. 49, 420-433 (1987)

28. Abdel-Gawad, HR, Thomas, DK: The Fekete-Szegö problem for strongly close-to-convex functions. Proc. Am. Math. Soc. 114(2), 345-349 (1992)

29. Darus, M, Thomas, DK: The Fekete-Szegö theorem for strongly close-to-convex functions. Sci. Math. 3(2), 201-212 (2000)

30. Choi, JH, Kim, YC, Sugawa, T: A general approach to the Fekete-Szegö problem. J. Math. Soc. Jpn. 59(3), 707-727 (2007)

31. Pommerenke, C: Univalent Functions. Vandenhoeck \& Ruprecht, Göttingen (1975)

32. Kowalczyk, B, Lecko, A: Fekete-Szegö problem for a certain subclass of close-to-convex functions. Bull. Malays. Math Soc. (accepted)

33. Laguerre, EN: Sur la théorie des équations numériques. J. Math. Pures Appl. 9, 99-146 (1883). In: Oeuvres de Laguerre, vol. 1, pp. 3-47, Paris (1898)

34. Jameson, GJO: Counting zeros of generalized polynomials: Descartes' rule of signs and Leguerre's extensions. Math. Gaz. 90(518), 223-234 (2006)

35. Turowicz, A: Geometria Zer Wielomianów (Geometry of Zeros of Polynomials). PWN, Warszawa (1967) (in Polish)

10.1186/1029-242X-2014-65

Cite this article as: Kowalczyk and Lecko: The Fekete-Szegö inequality for close-to-convex functions with respect to a certain starlike function dependent on a real parameter. Journal of Inequalities and Applications 2014, 2014:65

\section{Submit your manuscript to a SpringerOpen ${ }^{\ominus}$ journal and benefit from:}

- Convenient online submission

- Rigorous peer review

- Immediate publication on acceptance

- Open access: articles freely available online

- High visibility within the field

- Retaining the copyright to your article 